\title{
Quantificação da carga de jogos oficiais de futebol através do TRIMP
}

http://dx.doi.org/10.11606/1807-5509201800030351

\author{
Tane KANOPE* \\ Rodrigo Figueiredo MORANDI ${ }^{*}$ \\ Eduardo Mendonça PIMENTA*
}

*Escola de Educação

Física, Fisioterapia e

Terapia Ocupacional,

Universidade Federal

de Minas Gerais, Belo

Horizonte, MG, Brasil.

\section{Resumo}

A medida do impulso de treino (TRIMP) é um método amplamente utilizado para aferir a carga das sessões de treinamento de futebol. Entretanto, poucos estudos investigaram o TRIMP de jogos oficiais desta modalidade. 0 objetivo do estudo foi analisar e descrever o TRIMP de partidas oficiais de futebol. Dez jogadores ( $19 \pm 1,0$ anos; $0,3 \pm 7,2 \mathrm{~kg}, 181,3 \pm 6,6 \mathrm{~cm} ; 9,02 \pm 1,8 \%$ de gordura; $56,4 \pm 3,2 \mathrm{mLO} / \mathrm{kg} / \mathrm{min}-1$ ) da categoria sub 20 participaram do estudo durante três jogos oficiais. A frequência cardiaca máxima (FCmax) foi avaliada em teste de campo. A carga dos jogos foi mensurada através dos valores de TRIMP (TRIMPMOD e TRIMPB) e do percentual do tempo jogado nas cinco zonas de intensidade da FC, sendo desde a zona $1=65-71 \%$ da $F C$ max a 5=93-100\% da FCmax. 0 TRIMPMOD e TRIMPB mensurados foram, respectivamente, de 356,34 e 246,27, com diferença significativa entre eles $(p<0,05)$ e baixa correlação $(\rho=0,32)$. A intensidade de jogo encontrada foi equivalente a 87,76\%FCmax. A distribuição do tempo jogado em cada zona de intensidade foi: $Z 1=2,46 \%$, $Z 2=6,72 \%, Z 3=20,14 \%, Z 4=37,03 \%$ e $Z 5=33,65 \%$. Concluiu-se a diferença entre o TRIMPMOD e TRIMPB deve-se a sua forma de cálculo e que sempre o mesmo deve ser escolhido para monitoramento longitudinal da carga interna de jogos de futebol. Os atletas passaram a maior parte do tempo nas zonas de maior intensidade (Z4 e Z5). No segundo tempo houve um aumento significativo nas zonas 1 e 2 e uma diminuição nas zonas 4 e 5 , mostrando uma queda significativa da intensidade entre primeiro e segundo tempo.

PalavRas-chave: Impulso de treino; Frequência cardiaca; Esportes coletivos; Desempenho.

\section{Introdução}

A medida da frequência cardíaca (FC) é amplamente utilizada como método válido e de boa aplicabilidade para a estimativa da intensidade durante a pratica esportiva ${ }^{1,2}$. Seu uso como parâmetro para controle da intensidade se baseia na sua relação linear com o consumo de oxigênio $\left(\mathrm{VO}_{2}\right)$, mesmo em atividades intermitentes como o futebol ${ }^{3}$. Pelo fato da FC ser influenciada por diversos fatores como treinabilidade, gênero, condiçóes ambientais e estado de hidratação, Karvonen e VuorimaA ${ }^{4}$ recomendam que a FC seja expressa como percentual $\mathrm{da}$ frequência cardíaca máxima $\left(\% \mathrm{FC}_{\max }\right)$ para ser utilizada adequadamente no controle da intensidade em jogadores de futebol ${ }^{5,6}$.

Para aferir e monitorar a carga interna de sessōes de treinamento, BANISTER et al. ${ }^{7}$ propuseram um método denominado impulso de treino $\left(\right.$ TRIMP $_{B}$ ) calculado através da FC. Baseando-se que uma atividade física aumenta a FC entre seus valores de repouso e máximo, os autores propuseram que a resposta da FC de uma pessoa em decorrência do exercício, juntamente com a duração, poderia ser uma medida representativa da carga interna de treino. Porém, esse método, por utilizar apenas a FC média, pode apresentar limitaçóes, particularmente em tipos de exercícios intermitentes em que a resposta da FC é muito variável, contexto em que se encaixa o futebol ${ }^{8}$.

$\mathrm{Na}$ tentativa de criar um método capaz de quantificar adequadamente a carga interna de treino em esportes de caráter intermitente, STAGNo et al. ${ }^{8}$ propuseram modificaçóes do TRIMP originalmente proposto por BANISTER et al. ${ }^{7}$. Estas consistiram na divisáo da $\mathrm{FC}$ em cinco zonas de intensidade com base na FC correspondente às concentrações sanguíneas de lactato de 1,5 mmol.L-1 e 4,0 mmol.L-1. Esses valores foram escolhidos para que refletissem o perfil típico da curva representativa da resposta 
do lactato sanguíneo perante o aumento progressivo da intensidade. Dessa forma, o TRIMP modificado $\left(\right.$ TRIMP $_{\text {MOD }}$ ), proposto por STAGNO et al. ${ }^{8}$, apresentase como um modelo potencial para a mensuração da carga interna durante exercícios intermitentes.

De fato, diversos estudos utilizaram diferentes métodos de TRIMP para mensurar a carga interna de sessóes de treinamentos no futebol ${ }^{9-12}$. Entretanto, poucos estudos têm sido apresentados na literatura analisando essa variável durante os jogos oficiais ${ }^{13}$. A maioria dos estudos que utilizaram o TRIMP para mensurar a carga interna de partidas de futebol avaliaram jogos amistosos, jogos simulados ou jogos recreativos ${ }^{9,11}$. Além disso, não foram encontrados até o momento estudos que analisaram a dinâmica de jogos oficiais de futebol de acordo com o TRIMP MOD $_{\text {T }}$ e suas respectivas zonas de intensidade da FC.

O conhecimento mais detalhado da carga interna de uma partida oficial de futebol é de extrema importância já que se apresenta como referência para o aperfeiçoamento das sessôes de treinamentos ${ }^{14}$ e para sua relativização em relação à realidade competitiva. Dessa forma, considerando que o TRIMP é uma variável amplamente utilizada para aferir a carga interna em sessões de treinamentos no futebol ${ }^{9,11,12}$ e de que não existem informaçôes precisas a respeito de seu comportamento durante partidas oficiais, o presente estudo teve como objetivo analisar e descrever o TRIMP $_{\mathrm{B}}$ e TRIMP ${ }_{\text {MOD }}$ de jogos oficiais de futebol e suas respectivas zonas de intensidade da FC de acordo com o TRIMPMOD de STAGNO et al. ${ }^{8}$.

\section{Método}

\section{Participantes}

Participaram deste estudo dez jogadores da categoria sub 20 (dois zagueiros, dois laterais, três meio campistas e três atacantes) de um time da primeira divisão do futebol brasileiro. Todos participavam regularmente de sessóes de treinamento (duração média de 90 minutos, seis vezes por semana), competiam em eventos organizados pela Confederação Brasileira de Futebol e tinham em média $5 \pm 2$ anos de experiência na prática do futebol.

Esse estudo respeitou todas as normas estabelecidas pelo Conselho Nacional de Saúde (Resolução 466/12) envolvendo pesquisas com seres humanos e foi aprovado pelo Comitê de ética e pesquisa (COEP - 27609814.3.0000.5149). Previamente ao início do mesmo, os sujeitos foram informados dos possíveis riscos e benefícios, assinaram um termo de consentimento livre e esclarecido e foram instruídos de que poderiam deixar de participar a qualquer momento sem justificativas prévias. Para critério de inclusão, os atletas deveriam estar treinando regularmente há aproximadamente um mês e ter participado de pelo menos uma partida oficial completa. Como critério de exclusão, o atleta era desconsiderado do estudo caso fosse substituído durante a partida oficial monitorada ou interrompesse sua participação nos treinos/jogos em virtude de lesão ou outro motivo qualquer.

\section{Procedimentos}

Com o intuito de caracterizar a amostra foram mensuradas massa corporal, estatura, dobras cutâneas e consumo máximo de oxigênio $\left(\mathrm{VO}_{2 \max }\right)$. Para aferir a massa corporal utilizou-se uma balança digital (Welmy ${ }^{\oplus}$ ) com precisão de $0,01 \mathrm{~kg}$ previamente calibrada. A estatura foi medida utilizando-se um estadiômetro acoplado à balança digital com precisão de $0,1 \mathrm{~cm}$. As dobras cutâneas (subescapular, tríceptal, peitoral, axilar, suprailíaca, abdominal e da coxa) foram obtidas utilizando-se um plicômetro graduado em milímetros (Lange $\left.{ }^{\circ}\right)$. Os valores de cada dobra foram utilizados para a obtenção do somatório das dobras e, posteriormente, para o cálculo do percentual de gordura de acordo com a equação proposta por JACKSON e POLLOCK ${ }^{15}$.

$\mathrm{O} \mathrm{VO}_{2 \max }$ foi avaliado de forma indireta, por meio do teste de campo YoYo Endurance Test (nível 2) ${ }^{3}$, realizado uma semana antes do início da competição. Este é específico para o futebol e esportes intermitentes ${ }^{16}$ do qual a distância total percorrida tem relação positiva com a capacidade aeróbia dos atletas ${ }^{17}$.

Todos os voluntários foram monitorados por cardiofrequencímetros (Team System, Polar Electro $\mathrm{Oy}^{\oplus}$, Kempele, Finland) com uma taxa de amostragem de 5 em $5 \mathrm{~s}$. Estes eram entregues no vestiário por um dos pesquisadores 30 minutos antes do início da partida oficial/teste de campo. Ao término da atividade, os cardiofrequencímetros 
eram recolhidos pelo mesmo pesquisador para tratamento dos dados em software específico (Polar Precision Performance SW 3.0, Polar Electro Oy ${ }^{\odot}$ ). Esse sistema foi utilizado, pois permite a aferição da FC durante a atividade sem a necessidade de um monitor de pulso, o qual é proibido pela regulamentação do futebol. Durante a competição as condiçóes ambientais de temperatura e humidade relativa do ar $\left(32,86 \pm 1,62{ }^{\circ} \mathrm{C}\right.$ e $52,33 \pm 3,05 \%$, respectivamente) foram registradas através de um termo higrômetro digital (Instrutherm ${ }^{\bullet}$ HT-260). Os jogos ocorreram entre 14 e 16 horas, no mês de dezembro, na região sul do Brasil.

$\mathrm{A} \mathrm{FC}_{\text {max }}$ foi determinada através do maior valor de FC aferido durante o estudo, seja durante os jogos monitorados ou no teste de campo ${ }^{18}$. Posteriormente, ela foi usada para relativização individual da FC $\left(\% \mathrm{FC}_{\max }\right)$ como recomendado ${ }^{4,19}$. A carga interna dos jogos foi mensurada através dos valores de TRIMP e do percentual do tempo jogado nas cinco zonas da FC previamente descritas por STAGNo et al. ${ }^{8}$. A zona $1(\mathrm{Z} 1)$ corresponde a $65-71 \% \mathrm{FC}_{\text {max }}$, a zona 2 (Z2) a $72-78 \% \mathrm{FC}_{\text {max }}$, a zona $3(\mathrm{Z3})$ a $79-85 \% \mathrm{FC}_{\text {max }}$ a zona $4(\mathrm{Z} 4)$ a $86-92 \% \mathrm{FC}_{\max }$ e a zona $5(\mathrm{Z} 5)$ a $93-100 \% \mathrm{FC}_{\max }$.

O TRIMP ${ }_{\text {MOD }}$ e o TRIMP ${ }_{\text {B }}$ foram calculados através das seguintes fórmulas:

\section{Resultados}

As características dos sujeitos estáo apresentadas na TABELA 1.

A caracterização da intensidade de jogos oficiais da categoria sub 20 , através do $\% \mathrm{FC}_{\text {max }}$ TRIMP $_{\mathrm{B}}$, TRIMP $_{\text {MOD }}$ e do percentual do tempo jogado nas cinco zonas da FC está apresentada na TABELA 2. Ambos TRIMP $_{\text {MOD }}$ eTRIMP ${ }_{\mathrm{B}}$ obtidos no primeiro tempo $(184,4 \pm 23,9$ e 126,2 $\pm 13,4$, respectivamente) foram maiores em relação ao segundo $(165,5 \pm 25,8$ e $117,8 \pm 13,2$, respectivamente $)(\mathrm{p}<0,05) . \mathrm{Na}$ comparaçáo entre os métodos, o TRIMP ${ }_{\mathrm{B}}$ apresentou diferenças significativas em relação ao TRIMP ${ }_{\text {MOD }}$, tanto quando comparados os dois tempos da partida separadamente, quanto no valor total do jogo $(246,3 \pm 33,8$ e $356,3 \pm 56,3$, respectivamente $)$ $(\mathrm{p}<0,05)$ (FIGURA 1). Ainda, o TRIMP ${ }_{\text {MOD }}$ e TRIMP $_{B}$ apresentaram baixa correlação $(\rho=0,32)$. Com relação à dinâmica das partidas, a análise das diferentes zonas de intensidade mostrou que os jogos de futebol foram jogados na maior parte do tempo
TRIMP $_{\text {MOD }}=($ T Z1 11,25$)+(T Z 2 \cdot 1,71)+$

$(\mathrm{T} Z 3 \cdot 2,54)+(\mathrm{T} Z 4 \cdot 3,61)+(\mathrm{T} Z 5 \cdot 5,16)$

Onde $\mathrm{T}=$ Tempo jogado em cada zona ( $\mathrm{min})$.

$$
\text { TRIMP }_{\mathrm{B}}=\mathrm{T} \cdot \Delta \mathrm{FC} \cdot \mathrm{Y}
$$

Onde $\mathrm{T}=$ duraçấo total da atividade (min);

$\Delta \mathrm{FC}=\mathrm{FC}$ média de jogo $-\mathrm{FC}$ de repouso $/ \mathrm{FC}_{\max }-\mathrm{FC}$ repouso; $\mathrm{Y}=0,64 \mathrm{e}^{1,92 \mathrm{x}} ; \mathrm{e}=2,712 \mathrm{ex}=\Delta \mathrm{FC}$.

\section{Análise estatística}

Inicialmente, empregou-se a estatística descritiva com a média e desvio padrão (DP) para a caracterização da amostra e da carga de jogos de futebol. Posteriormente foi realizado o teste de Shapiro Wilk, verificando-se uma distribuição assimétrica dos dados. A correlação entre os TRIMPs foi verificada através do coeficiente de correlação de Spearman ( $\rho)$. A seguir, a análise de variância de Fridman seguida do teste de Wilcoxon foi utilizada para comparaçóes múltiplas entre o TRIMP $_{\text {MOD }}$ e TRIMP ${ }_{\text {B }}$ e entre as zonas da FC.

Todas as análises estatísticas foram realizadas através dos softwares Statistical Package for the Social Sciences ${ }^{\oplus}$ (SPSS, EUA) e Microsoft Excel $^{\oplus}$ para Microsoft Windows 10 (Microsoft $\left.{ }^{\oplus}, \mathrm{EUA}\right) \mathrm{e}$ $\mathrm{o}$ nivel de significância adotado foi de $\mathrm{p}<0,05$.

na zona 4, com os percentuais de tempo jogado nas zonas 4 e 5 significativamente maiores do que nas zonas 1,2 e $3(\mathrm{p}<0,05)$ (FIGURA 2).Analisando mais detalhadamente, identificou-se que o percentual do tempo jogado na $\mathrm{Z} 1$ e Z2 foram maiores no segundo tempo $(4,2 \pm 3,7$ e $11 \pm 8,5 \%$, respectivamente) quando comparado com o primeiro tempo de jogo $(1,3 \pm 2,9$ e $3,6 \pm 7,1 \%$, respectivamente $)$ $(\mathrm{p}<0,05)$. Por outro lado, o percentual do tempo jogado na Z4 e Z5 foram maiores no primeiro tempo $(39,1 \pm 13,4$ e $35 \pm 19,1 \%$, respectivamente) em relação ao segundo tempo $(32,7 \pm 13,5$ e $29,2 \pm 18,9 \%$, respectivamente) $(p<0,05)$. Não foi observada diferença significativa entre primeiro $(20,8 \pm 10,2 \%)$ e segundo tempo $(23 \pm 10,5 \%)$ (p>0,05) na Z3 (FIGURA 3).

Analisando mais detalhadamente, identificouse que o percentual do tempo jogado na $\mathrm{Z} 1$ e $\mathrm{Z} 2$ foram maiores no segundo tempo $(4,2 \pm 3,7 \mathrm{e}$ $11 \pm 8,5 \%$, respectivamente) quando comparado 
com o primeiro tempo de jogo $(1,3 \pm 2,9$ e 3,6 $\pm 7,1 \%$, respectivamente $)(p<0,05)$. Por outro lado, o percentual do tempo jogado na Z4 e Z5 foram maiores no primeiro tempo $(39,1 \pm 13,4$ e $35 \pm 19,1 \%$, respectivamente $)$ em relação ao segundo tempo $(32,7 \pm 13,5$ e $29,2 \pm 18,9 \%$, respectivamente) $(p<0,05)$. Não foi observada diferença significativa entre primeiro $(20,8 \pm 10,2 \%)$ e segundo tempo $(23 \pm 10,5 \%)$ ( $>0,05)$ na Z3 (FIGURA 3).

TABELA 1 - Caracterização da amostra.

\begin{tabular}{cccccc}
\hline $\mathbf{N}$ & $\begin{array}{c}\text { Idade } \\
(\mathbf{a n o s})\end{array}$ & $\begin{array}{c}\text { Massa Corporal } \\
(\mathbf{k g})\end{array}$ & $\begin{array}{c}\text { Estatura } \\
(\mathbf{c m})\end{array}$ & \%Gordura & $\begin{array}{c}\text { VO }_{2 \max } \\
\left(\mathbf{m L O}_{2} \cdot \mathbf{k g}^{-1} \cdot \mathbf{m i n}^{-1}\right)\end{array}$ \\
\hline 10 & $19 \pm 1,00$ & $70,3 \pm 7,21$ & $181,3 \pm 6,63$ & $9,02 \pm 1,82$ & $56,4 \pm 3,20$ \\
\hline
\end{tabular}

TABELA 2 - Intensidade de jogos de futebol.

\begin{tabular}{lcccccccc}
\hline & \%FC $_{\max }$ & TRIMP $_{\mathrm{B}}$ & TRIMP $_{\text {MOD }}$ & Z1(\%) & Z2(\%) & Z3(\%) & Z4(\%) & Z5(\%) \\
\hline Média & 87,76 & 246,27 & 356,34 & 2,46 & 6,72 & 20,14 & 37,03 & 33,65 \\
DP & 2,07 & 33,79 & 56,33 & 3,31 & 7,49 & 11,42 & 13,60 & 20,95 \\
\hline
\end{tabular}

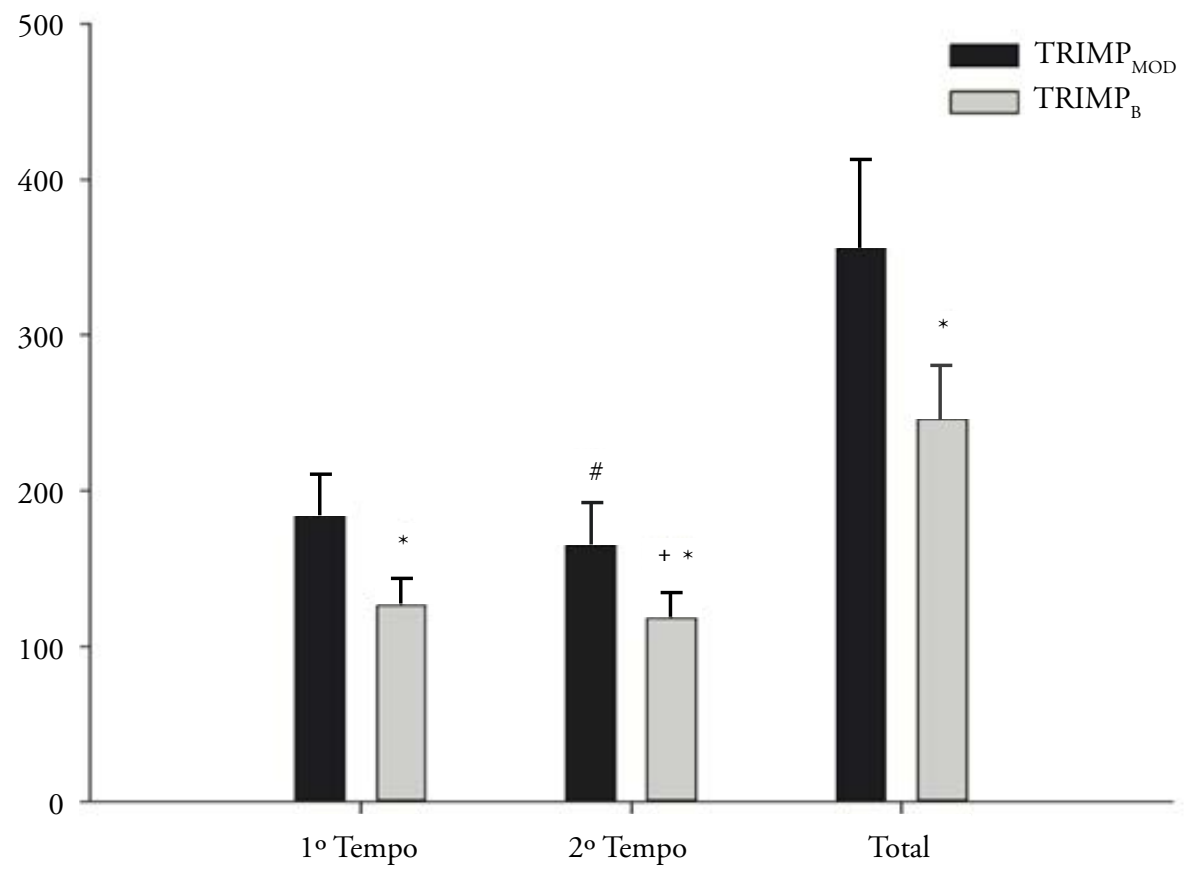

FIGURA 1 - Valores do TRIMP ${ }_{\mathrm{MOD}}$ e TRIMP ${ }_{\mathrm{B}}$. ${ }^{*}$ Estatisticamente significativo em relação ao TRIMPMOD em uma mesma faixa temporal. "Estatisticamente significativo em relação ao $1^{0}$ tempo. ${ }^{+}$Estatisticamente significativo em relação ao $1^{0}$ tempo. Para todas as diferenças o p<0,05. 


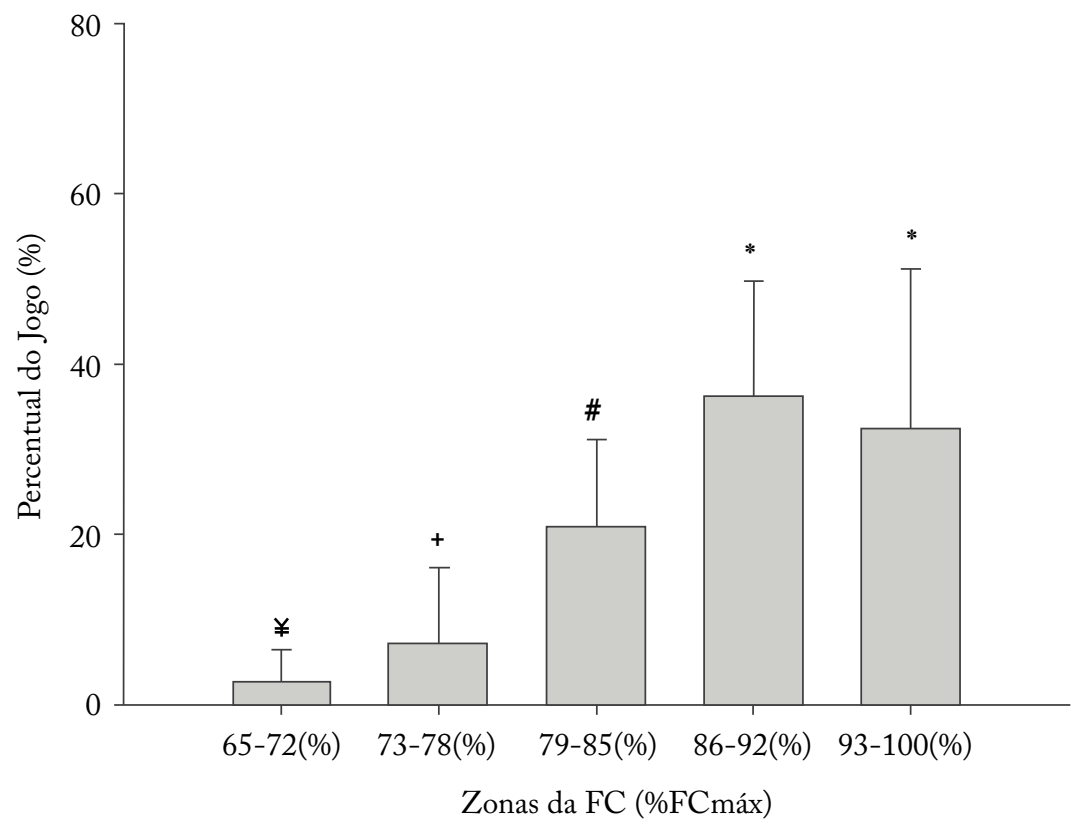

FIGURA 2 - Intensidade de jogos de futebol expressa em percentual do tempo jogado nas diferentes zonas de intensidade. *Estatisticamente significativo em relação a Z1, Z2 e Z3. "Estatisticamente significativo em relação a Z1, Z2, Z4 e Z5. ${ }^{+}$Estatisticamente significativo em relação a Z1, Z3, Z4 e Z5. ${ }^{\mathrm{Z}}$ Estatisticamente significativo em relação a Z2, Z3, Z4 e Z5. Para todas as diferenças o p <0,05.

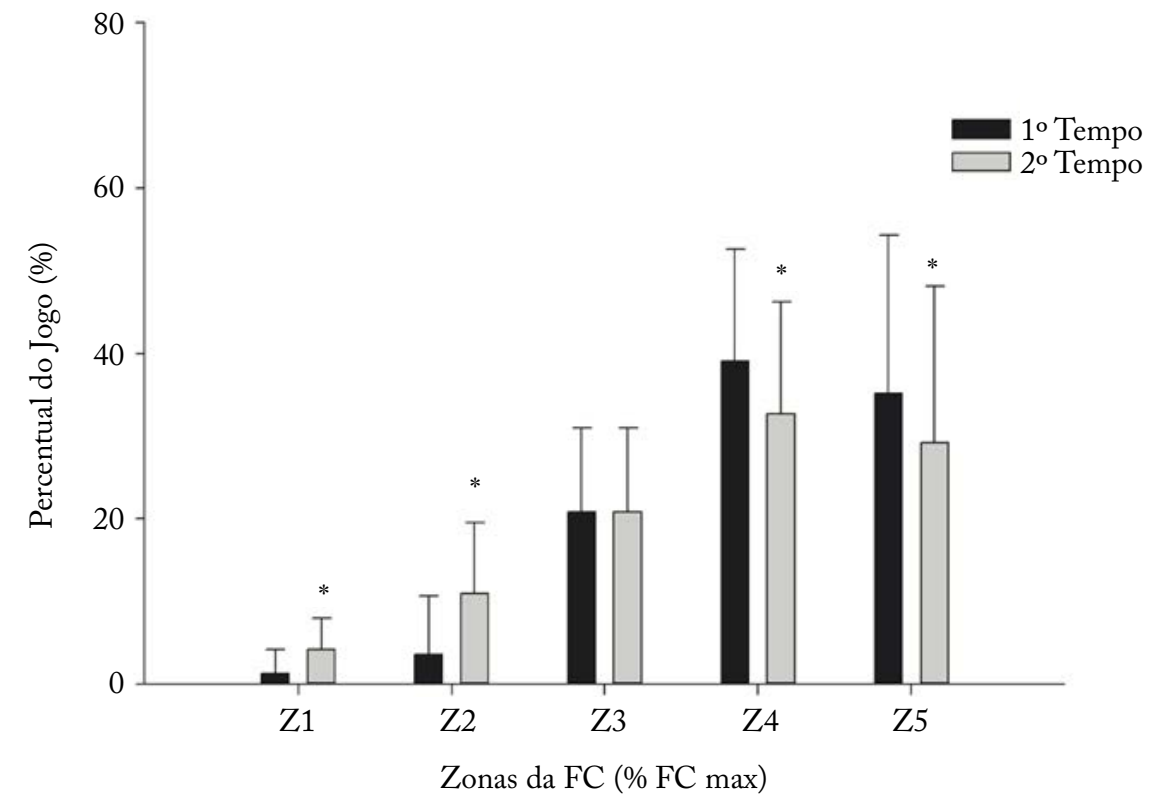

FIGURA 3 - Comparação do percentual do tempo de jogo em diferentes zonas de intensidade entre primeiro e segundo tempo. *Estatisticamente significativo em relação ao primeiro tempo na mesma zona de intensidade $(\mathrm{p}<0,05)$. 


\section{Discussão}

O objetivo do presente estudo foi analisar e descrever o TRIMP de jogos oficiais de futebol através do TRIMP ${ }_{B}$, TRIMP $_{\text {MOD }}$ e das zonas de intensidade da FC de acordo com StaGno et al. ${ }^{8}$. O valor total do TRIMP e TRIMP $_{\text {MOD }}$ encontrado foi de 246,67 e 356,34, respectivamente (TABELA 2). Sabendo-se como comportam as variáveis monitoradas ao longo das sessóes de treinos durante as competiçóes oficiais, permite aos treinadores manipularem suas sessōes de treino no intuito de aproximá-las cada vez mais das situaçóes competitivas. Dessa forma, mesmo considerando que os TRIMPs, por serem derivados da FC, sofram influência de fatores climáticos, relativos à hidratação e estado emocional ${ }^{4}$, o presente achado permite aos treinadores um referencial para suas sessóes de treinamento, cabendo a eles não somente manipularem seus treinos o máximo possível para maior representatividade de uma partida competitiva, mas para também poderem relativizar suas sessóes com maior segurança em relação a um jogo oficial.

O valor total do TRIMP $_{\mathrm{B}}$ e TRIMP ${ }_{\text {MOD }}$ encontrado foi de 246,67 e 356,34, respectivamente (TABELA 2). BARA FilHo et al. ${ }^{11}$ encontraram valores de TRIMP inferiores para jogos amistosos e simulados (249,91 e 205,83, respectivamente) em uma amostra de 30 atletas de uma equipe profissional da primeira divisão do campeonato mineiro. Confrontando os resultados, observa-se que, possivelmente, o caráter competitivo de jogos oficiais resultou em maior carga interna quando comparados com jogos amistosos, simulados ou recreativos. Até o presente momento, não foram localizados nas bases Web of Science, Medline e SciELO, estudos que utilizaram o TRIMP $_{\mathrm{B}}$ ou TRIMP ${ }_{\text {MOD }}$ e as zonas da FC propostas por STAGNo et al. ${ }^{8}$ para aferir a intensidade e a dinâmica de jogos oficiais de futebol. Possivelmente, pela dificuldade de coleta com relação ao equipamento, necessidade de pessoal especializado e restrições de uso impostas pela federação da modalidade, justifica-se essa escassez de estudos nesse contexto. Isso dificultou a análise e discussão dos dados aqui apresentados.

Foram observadas diferenças significativas entre o TRIMP ${ }_{\text {MOD }}$ e o TRIMP ${ }_{\mathrm{B}}$ (FIGURA 1) e a correlação entre eles foi considerada baixa $(\rho=0,32)$. Apesar de ambos serem considerados medidas representativas da carga interna da sessão de treinamento ${ }^{20,21}$, já que levam em consideração para o seu cálculo tanto a intensidade quanto a duraçáo do exercício, esses achados podem ser explicados pelo método de obtenção de cada um deles. $\mathrm{O}_{\text {TRIMP }}{ }_{\mathrm{B}}$ é calculado levando-se em conta apenas a FC média da atividade. Portanto, em modalidades intermitentes, pode apresentar limitações ${ }^{21}$. Já o TRIMP $_{\text {MOD }}$ leva em conta diferentes zonas de FC em função da concentração sanguínea de lactato, o que lhe torna mais indicado para atividades intermitentes. Além disso, o método proposto por STAGNo et al. ${ }^{8}$ permite uma análise do tempo jogado em cada zona de intensidade da FC, o que possibilita não só uma avaliação da intensidade, mas também informaçóes a respeito da dinâmica de jogos e treinamentos. Entretanto, vale ressaltar que, para isso, necessita de um software mais avançado que permita as configuraçóes das zonas de FC, o que nem sempre pode ser acessível. Dessa forma, deve-se prevalecer o custo benefício e sabendo da diferença entre ambos, adotar sempre o mesmo método para realização de controle e monitoramento longitudinal das sessóes de treinamentos.

A intensidade do jogo observada através do $\% \mathrm{FC}_{\max }$ no presente estudo $\left(87,76 \% \mathrm{FC}_{\max }\right)$ foi similar aos achados de Tumilty et al. ${ }^{22}\left(87 \% \mathrm{FC}_{\max }\right)$ que avaliaram 16 jogadores de futebol de um time australiano sub 20. Na FIGURA 2, observou-se que os atletas jogaram a maior parte do tempo nas zonas de intensidade mais elevadas (Z4 e Z5, respectivamente). Esses dados diferem dos estudos de Coelho et al. ${ }^{19}$ e Helgerud et al. ${ }^{23}$, do qual os autores relataram valores percentuais mais altos em zonas de menor intensidade e valores mais baixos na zona de maior intensidade. Os dados apresentados nos estudos supracitados mostram que os atletas jogaram a maior parte do tempo nas faixas de 70 a $85 \%$ e 85 a $90 \%$ da $\mathrm{FC}_{\text {max }}$, que correspondem respectivamente às zonas $2,3 \mathrm{e} 4 \mathrm{do}$ presente trabalho.

Particularmente, no presente estudo, o tempo jogado na Z5 representou 33,65\% do tempo total de jogo. Coelho et al. ${ }^{19}$ apesar de terem utilizado uma metodologia diferente para a divisão das zonas de intensidade da $\mathrm{FC}^{23}$, encontraram valores inferiores $(9,10 \%)$ para o tempo jogado na zona de maior intensidade $\left(95-100 \% \mathrm{FC}_{\max }\right)$. Como visto por ANTONACCI et al. ${ }^{18}$, utilizar testes para determinar a $\mathrm{FC}_{\max }$ pode subestimá-la quando comparado com jogos oficiais, visto o seu caráter pouco motivador. Dessa forma, como o presente estudo e o do Coelho et al. ${ }^{19}$ utilizaram uma metodologia semelhante para obtenção da $\mathrm{FC}_{\max }$, 
uma possível explicação para essa diferença pode estar relacionada à menor intensidade dos jogos observada por Coelho et al. ${ }^{19}\left(\approx 84 \% \mathrm{FC}_{\max }\right)$ com a aqui apresentada $\left(88 \% \mathrm{FC}_{\max }\right)$. Corroborando ainda mais com este ponto de vista, deve-se atentar que a intensidade dos jogos apresentada por Coelho et al. ${ }^{19}$ foi inferior à intensidade do limiar de lactato $\left(\approx 87 \% \mathrm{FC}_{\text {max }}\right)$ de seus atletas.

Durante o segundo tempo de um jogo de futebol é esperado uma redução da intensidade. Fatores relacionados à fadiga podem estar associados com essa redução ${ }^{24}$. Uma possível explicação fisiológica para a queda no desempenho pode estar atrelada a depleção do glicogênio muscular que ocorre durante uma partida de futebol, o que especificamente no segundo tempo, limita a capacidade do atleta em manter atividades em alta intensidade ${ }^{3}$. Entretanto, outros fatores como o resultado parcial do jogo e a posição que o time se encontra na tabela de classificaçáo do campeonato, também podem influenciar na intensidade de uma partida de futebol. Nesse contexto, no presente estudo foi observada uma queda na intensidade de jogo no segundo tempo em relação ao primeiro. Vale ressaltar que, os jogos aqui analisados ocorreram durante a fase classificatória do campeonato e o time obteve uma derrota no primeiro jogo e duas vitórias nos jogos seguintes. Nas vitórias alcançadas pela equipe, o resultado foi estabelecido ainda no primeiro tempo dos jogos, o que pode ter influenciado na queda da intensidade. Esta, pode ser melhor visualizada ao se comparar os percentuais de tempo jogado nas zonas de baixa (Z1 e Z2) e alta intensidade (Z4 e Z5) (FIGURA 3), refletindo, inclusive, em TRIMP ${ }_{\mathrm{MOD}}$ significativamente diferentes $(184,4 \pm 23,9$ e $165,5 \pm 25,8$, respectivamente) (FIGURA 1). Esses dados corroboram com outros estudos que mostraram uma queda similar ${ }^{19,22,25}$, mesmo utilizando diferentes zonas de intensidade da $\mathrm{FC}^{14}$.

Uma das limitações do presente trabalho é o monitoramento de atletas da categoria sub 20 durantes três partidas oficiais de um campeonato a nível nacional. Dessa forma, seus resultados não devem ser extrapolados para atletas de diferentes categorias. Por se tratar de jogos oficiais, o estado de desidratação não pode ser controlado. Sabendose que a intensidade de um jogo de futebol, dentre outros fatores, pode ser influenciada pelo adversário e pelo estilo de jogo imposto pelo treinador da equipe, o número de jogos e números de atletas observados, devem ser levados em consideraçáo na interpretação dos resultados apresentados.

A partir dos dados encontrados no presente estudo, concluiu-se que o TRIMP ${ }_{\text {MOD }}$ apresentou baixa correlação e diferença significativa em relação ao TRIMP ${ }_{B}$. Portanto, sempre o mesmo TRIMP deve ser utilizado para monitoramento longitudinal da carga interna das sessóes de treinamento. Entretanto, independente do TRIMP utilizado, todos são calculados levando-se em conta a FC. Dessa forma, esse parâmetro pode apresentar limitaçóes em alguns momentos de atividades que possuem caráter intermitente, como é o caso do futebol que possui contribuição tanto aeróbica quanto anaeróbica. Nas partidas avaliadas, os atletas passaram a maior parte de tempo jogado na Z4 $\left(86-92 \% \mathrm{FC}_{\max }\right)$ e Z5 (93-100\%FCm $\mathrm{ax}_{\mathrm{ax}}$. Houve uma queda nos percentuais de tempo jogado nas zonas 4 e 5 em um aumento do tempo gasto nas zonas 1 e 2 entre primeiro e segundo tempo, caracterizando uma queda da intensidade na segunda metade do jogo.

\section{Abstract}

\section{Quantification of Internal Load (TRIMP) in Official Soccer Games}

The measurement of the training impulse (TRIMP) is a widely used method to measure the load of football training sessions. However, few studies have investigated the TRIMP of official games of this modality. The aim of the study was to analyze and describe the TRIMP of official soccer games. Ten under-20 soccer players ( $19 \pm 1.0$ years; $70.3 \pm 7.2 \mathrm{~kg}, 181.3 \pm 6.6 \mathrm{~cm} ; 9.02 \pm 1.8 \%$ body fat; $56.4 \pm 3.2 \mathrm{mLO} / \mathrm{kg} / \mathrm{min}-1)$ participated in the study during three official games. The maximum heart rate $\left(\mathrm{HR}_{\max }\right)$ was assessed by a field test. The game load was measured through TRIMP values $\left(T_{R} I M P_{M O D}\right.$ and TRIMP $\left.{ }_{B}\right)$ and the percentage of time played in the five intensity zones raginng from zone $1=65-71 \% \mathrm{HRmax}$ to $5=93-100 \% \mathrm{HR}_{\max }$. The game intensity found was equivalent to $87.76 \% \mathrm{HR}_{\text {max }}$.The measured TRIMPMOD and TRIMPB were, respectively, 356.34 and 246.27 . Both were significantly different $(p<0.05)$ and showed low correlation between them $(\rho=0,32)$. The game intensity was equivalent to $87.76 \% \mathrm{HR}_{\max }$. The distribution of the time played in each intensity zone was: $Z 1=2.46 \%, Z 2=6.72 \%, Z 3=20.14 \%, Z 4=37.03 \%$ and $Z 5=33.65 \%$. It was concluded that the differences between the TRIMP ${ }_{\text {MOD }}$ and TRIMP ${ }_{B}$ was due to their specific calculation 
norms and that the same method must be chosen for longitudinal monitoring of the internal load of soccer games. The athletes spent the most time in the higher intensity zones (Z4 and Z5). In the second half there was a significant increase in zones 1 and 2 and a decrease in zones 4 and 5, showing a significant drop in intensity between the first and second half.

KEYWORDS: Training impulse; Heart rate; Collective sports; Performance.

\section{Referências}

1. Capranica L, Tessitore A, Guidetti L, Figura F. Heart rate and match analysis in pre-pubescent soccer players. J Sports Sci 2001;19(6):379-84.

2. Esposito F, Impellizzeri FM, Margonato V, Vanni R, Pizzini G, Veicsteinar A. Validity of heart rate as an indicator of aerobic demand during soccer activities in amateur soccer players. Eur J Appl Physiol 2004;93(1-2):167-72.

3. Bangsbo J. The physiology of soccer: with special reference to intense intermittent exercise. Acta Physiol Scand Suppl. 1994;619:1-155.

4. Karvonen J, Vuorimaa T. Heart hate and exercise intensity during sports activities: practical application. Sports Med. 1988;5(5):303-11.

5. Achten J, Jeukendrup AE. Heart hate monitoring: application and limitations. Sports Med. 2003;33(7):517-38.

6. Impellizzeri F, Rampinini E, Marcora SM. Physiological assessment of aerobic training in soccer. J Sports Sci. 2005;23(6):583-92.

7. Banister E. Modeling elite athletic performance. In: MacDougall JD, Wenger HA, Green HJ. Physiological testing of the high-performance athlete. 2. ed. Champaign: Human Kinetics; 1991. p.403-25.

8. Stagno KM, Thatcher R, van Someren KA. A modified TRIMP to quantify the in-season training load of team sport players. J Sports Sci. 2007;25(6):629-34.

9. Campos-Vazquez MA, Mendez-Villanueva A, Gonzales-Jurado JA, León-Prados JA, Santalla A, Suarez-Arrones L. Relationships between rating-of-perceived-exertion- and heart-rate-derived internal training load in professional soccer players: a comparison of on-field integrated training sessions. Int J Sports Physiol Perform. 2015;10(5):587-92.

10. Bara-Filho MG, Freitas DS, Moreira D, Matta MO, Lima JRP, Nakamura FY. Heart rate variability and soccer training: a case study. Motriz: Rev Educ Fis. 2013;19(1):171-77.

11. Bara-Filho M, Matta M, Freitas DS, Miloski B. Quantificação da carga de diferentes tipos de treinamento no futebol. J Phys Educ. 2011;22(2):239-46.

12. Freitas DGS, Pinto A, Damasceno VO, Freitas VH, Miloski B, Bara Filho MG. Efeitos do treinamento sobre variáveis psicofisiológicas na pré-temporada de futebol. Rev Bras Med Esporte. 2014;20(1):26-31.

13. Rebelo A, Brito J, Seabra A, Oliveira J, Drust B, Krustrup P. A new tool to measure training load in soccer training and match play. Int J Sports Med. 2012;33(4):297-304.

14. Coelho DB, Rodrigues VM, Condessa LA, Mortimer LACF, Soares DD, Silami-Garcia E. Intensidade de sessóes de treinamento e jogos oficiais de futebol. Rev Bras Educ Fís Esp. 2008;22(3):211-8.

15. Jackson AS, Pollock ML. Generalized equations for predicting body density of men. Br J Nutr. 1978;40(3):497-504.

16. Krustrup P, Mohr M, Amstrup T, Rysgaard T, Johansen J, Steensberg A, et al. The yo-yo intermittent recovery test: physiological response, reliability and validity. Med Sci Sports Exerc. 2003;35(4):697-705.

17. Castagna C, Impellizzeri FM, Chamari K, Carlomagno D, Rampinini E. Aerobic fitness and yo-yo continuous and intermittent tests performances in soccer players: a correlation study. J Strength Cond Res. 2006;20(2):320-5.

18. Antonacci L, Mortimer LF, Rodrigues VM, Coelho DB, Soares DD, Silami-Garcia E. Competition, estimated, and test maximum heart hate. J Sports Med Phys Fitness. 2007;47(4):418-21.

19. Coelho DB, Mortimer LA, Condessa LA, Morandi RF, Oliveira BA, Marins JCB, et al. Intensity of real competitive soccer matches and differences among player positions. Rev Bras Cineantropom Desempenho Hum. 2011;13(5):341-7.

20. Borresen J, Lmabert MI. The quantification of training load, the training response and the effect on performance. Sports Med. 2009;39(9):779-95.

21. Nakamura FY, Soares-Caldeira LF, Okuno NM, Moreira A, Simões HG. Métodos de quantificação de carga de treinamento em exercício realizado no máximo estado estável de lactato. Motriz: Rev Educ Fis. 2010;16(2):1-9. 
22. Tumilty D. Physiological characteristics of elite soccer players. Sports Med. 1993;16(2):80-96.

23. Helgerud J, Engen LC, Wisloff U, Hoff J. Aerobic endurance training improves soccer performance. Med Sci Sports Exerc. 2001;33(11):1925-31.

24. Rienzi E, Drust B, Reilly T, Carter JE, Martin A. Investigation of anthropometric and work-rate profiles of elite South American international soccer players. J Sports Med Phys Fitness. 2000;40(2):162-9.

25. Coelho DB, Coelho LGM, Morandi RF, Ferreira Junior JB, Marins JCB, Prado LS, et al. Effect of player substitutions on the intensity of second-half soccer match play. Rev Bras Cineantropom Desempenho Hum. 2012;14(2):183-91.

\begin{tabular}{r|l} 
ENDEREçO & Recebido para publicação: 26/04/2016 \\
Tane Kanope & Revisado: 13/08/2016 \\
Av. Otacílio Negrão de Lima, 7100, Pampulha & Aceito: 19/10/2016 \\
31340-380 - Belo Horizonte - MG - BRASIL & \\
E-mail: tane.ufrj@gmail.com & \\
\hline
\end{tabular}

